\section{Reflections on the future of swarm robotics}

\author{
Marco Dorigo ${ }^{1 *}$, Guy Theraulaz ${ }^{2,3}$, Vito Trianni ${ }^{4 *}$ \\ Swarm robotics will tackle real-world applications by leveraging automatic design, heterogeneity, and hierarchical \\ self-organization.
}

The news is full of stories about robots conquering new grounds-a new application, a new operation environment, or a new level of performance. With rare exceptions, these stories are mostly about one or a few robots working together. In fact, the deployment of large groups of robots, or robot swarms, that coordinate and cooperatively solve a problem or perform a task, remains a challenge.

For the past two decades, overcoming this challenge has been the goal of swarm robotics, a research field that takes inspiration from natural self-organizing systems such as social insects, fish schools, or bird flocks and attempts to recreate the emergence of collective behavior from simple local interaction rules. Its goal is to make multi-robot systems more robust, fault-tolerant, and flexible than single robots.

The swarm robotics field finds its roots in a few seminal works published in the 1990s $(1,2)$, but it started to grow substantially only after the year 2000, moving from a small domain featuring studies with a clear biological inspiration to a mature research field involving many laboratories and researchers worldwide.

Nowadays, robot swarms have been demonstrated in lab settings, frequently employing a small number of robots with compact size $(3,4)$. Although technological innovations are pushing boundaries toward ever smaller scales $(5,6)$ and larger numbers $(7,8)$, the path toward real-word applications is still long and arduous. Future robot swarms will exist and operate at many different scales: physical scale-from micro/nano robots to large ground, aerial, and aquatic robots; group scale-from a few dozen to millions of individuals composing the swarm; temporal scalefrom swarms that display fast intervention and quick adaptation in a rapidly evolving environment to robots that continuously operate on months-long missions (e.g., on a distant planet); and spatial scale-from deployments in small confined spaces to missions across expansive unbounded fields. To design and manage such a wide range of possible systems, the key challenge will be to define a rigorous engineering methodology to program the individual robots so that the swarm as a whole acts as desired. This is no easy task, because the characteristics of swarms at different scales might require radically different approaches.

To grasp the variety of possible systems and applications, we suggest a few general design principles that remain valid across scales. First, the increasing complexity of swarm systems is such that their design cannot be accomplished solely by traditional approaches. The more robot swarms will be confronted with uncertain/unpredictable environments and will rely on intricate patterns of interactions, the more automated design methodologies will be necessary to obtain desired behaviors, because they can be employed to generate individual rules that are evaluated for their effects on swarm performance (9). Machine learning with data-driven approaches becomes relevant whenever model-based solutions are too demanding, for instance, when it is difficult to provide a precise model of robot-environment interactions (e.g., due to complex physical interactions among micro/nano robots or unpredictable environmental dynamics as caused by underwater currents). To address selforganized control, the learned control architectures need to suitably integrate robot perceptions with information asynchronously received from (possibly hundreds of) peers and with memory of past states. Hybrid systems mixing model-free and model-based approaches will likely provide additional power, learning from data those aspects that are peculiar to the problem at hand (e.g., the

\footnotetext{
${ }^{1}$ Institut de Recherches Interdisciplinaires et de Développements en Intelligence Artificielle (IRIDIA), Université Libre de Bruxelles (ULB), Brussels, Belgium. ${ }^{2}$ Centre de Recherches sur la Cognition Animale (CRCA), Centre de Biologie Intégrative (CBI), Université de Toulouse, CNRS, UPS, Toulouse, France. ${ }^{3}$ Centre for Ecological Sciences, Indian Institute of Science, Bengaluru, India. ${ }^{4}$ Institute of Cognitive Sciences and Technologies (ISTC), National Research Council (CNR), Rome, Italy.

*Corresponding author. Email: mdorigo@ulb.ac.be (M.D.); vito.trianni@istc.cnr.it (V.T.)
}

response of a noisy communication medium characterizing the target application). Overall, automated methods hold the potential to liberate the designer from tedious trial and error or parameter tuning and to better deal with the specific swarm scales required by the task, resulting in a general engineering methodology that can be suitably applied across different application domains.

Second, in order for robot swarms to perform ever more complex tasks, they will likely need to be heterogeneous, both in hardware features and in roles that individual robots can play within the swarm (4). Physical heterogeneity can equip the robot swarm for tasks that require different hardware (e.g., fast moving drones for collaborative monitoring that cooperate with slow ground robots capable of modifying the environment). Behavioral heterogeneity can enable specialization (possibly via local learning mechanisms) and can underlie phase transitions that make the emergent collective behavior more flexible, adaptive to new conditions, and resilient to external perturbations. By abandoning the traditional homogeneity of hardware and control, flexibility and autonomy can be improved for small and large swarms, and operations can be expanded to address a wider spectrum of spatial and temporal scales. The additional dimensions introduced by heterogeneity (i.e., number of different roles and their proportion within the swarm) entail increased complexity in the design and further motivate the exploitation of automated techniques.

Last, robot swarms should include mechanisms to allow hierarchical forms of control beyond traditional pure self-organization. Whereas the latter features a flat organizational structure, a hierarchical control approach assigns a few individuals larger responsibilities, flexibly adapting the hierarchy to the task execution demands (10). In this way, it could be possible to more efficiently address task allocation, creation of task-oriented teams within the swarm, coordination of specific activities, or interaction with human users. 
Table 1. Timeline of swarm robotics research. Milestones reached by swarm robotics research in the past and projections of future developments. For each milestone, we indicate whether it entails innovation in software (SW), hardware (HW), or both.

A new paradigm is tested in which collaboration is emergent from simple (often bioinspired)
behaviors. First experiments with robots demonstrating self-organization by means of indirect (stigmergic) and local interactions, with clear inspiration from swarm intelligence. manipulation of objects, task allocation, and tasks that strictly require collaboration in order to be solved.

2002-2006

2004-2008

2005-2009

2006-2010

2010-2015

2014-2019

2016-2020

2020-2025

2020-2030

2025-2030

2025-2035

2030-2040

2030-2045

2035-2050
The Swarm-bots project demonstrates robot swarms capable of self-assembly. Robots are capable of building pulling chains and large structures that can transport heavy weights and deal with rough terrain.

Initial demonstrations of the automatic design of robot swarms by means of evolutionary algorithms, leading to the establishment of the evolutionary swarm robotics approach.

First attempts at developing standard swarm robotics platforms (e-pucks) and miniature robots for swarm robotics research (Alice, Jasmine).

The Swarmanoid project demonstrates for the first time heterogeneous robot swarms composed of three groups of robots: flying, climbing, and ground-based robots.

Different approaches appear for the design of robot swarms: Advanced methods for automatic design (AutoMoDe, novelty search), design patterns, mean-field models, and optimal stochastic approaches.

The "control without computation" approach develops swarm robotics behaviors with direct sensor-actuator mapping and no computation whatsoever.

Swarms of flying drones become available for research, and decentralized solutions are studied and deployed.

First demonstration of robot swarms capable of autonomously learning a suitable collective behavior for a given class of problems.

First civil applications of robot swarms to precision agriculture and infrastructure inspection and maintenance. Military applications largely use non-combat unmanned drones to cooperatively accomplish information gathering and mission support actions.

Deployment of robot swarms for maritime and deep-sea applications, providing support to ecological monitoring, surveillance, and fishing.

The entertainment sector uses robot swarms for interactive, immersive displays. Robot swarms are employed within the city, sharing the environment with operators and citizens. Robots will be insect- or pet-like devices that will collaborate to carry out service tasks such as cleaning, grazing, or delivering goods.

First space exploration mission on the Moon and Mars with miniature rover swarms, expanding the explored area and demonstrating on-site construction abilities.

Millimeter-scale soft-bodied robot swarms enter agricultural fields for pest control or aquatic environments to collect microplastics.

Microscopic robot swarms are demonstrated for medical applications such as targeted drug delivery, and clinical trials with human participants begin.
SW

HW and SW

SW

HW

HW and SW

SW

SW

$\mathrm{HW}$ and SW

SW

$H W$ and SW

$H W$

$H W$ and SW

$H W$

$H W$ and $S W$

HW and SW
Such hierarchies should, however, not be imposed from the outside. Rather, they should themselves be the result of self-organizing processes where some robots in the swarm may take leading roles depending on their specific characteristics, on the task being performed, and on the environmental conditions in which they are placed. In this respect, leveraging heterogeneity for robot swarm control-as advocated above-becomes even more consequential.

An (automated) engineering methodology for heterogeneous, hierarchically self-organized robot swarms is still missing but is crucially needed to successfully transition from laboratories to real-world applications (see
Table 1). We believe that the first deployments of these unconventional robot swarms will be in precision agriculture, infrastructure inspection and maintenance, and noncombat military applications. Precision agriculture features expansive fields where different types of robots can operate in parallel and can collaborate in heterogeneous teams to perform important actions such as weeding, fertilizing, or harvesting. Infrastructure inspection can benefit from the parallel operation carried out by large numbers of small robots exploring different areas and collectively identifying the presence of faults or damage. Non-combat defense operations will benefit from swarms that-by exploiting hierarchical self-organizationare difficult to shut down because they can flexibly re-organize and self-repair. Later in the next decade, aquatic surface and underwater robots will be mature enough to be deployed at sea, carrying out inspection, ecological monitoring, deep-sea exploration, and sustainable fishing. Robot swarms will then likely enter our cities, taking over service and entertainment tasks in a coordinated and self-organized way, while interacting with human workers and citizens. The use of robot swarms in space missions could come next and could play a key role in large-scale exploration of the Moon and Mars by 2040 . 
Another possible future direction takes advantage of miniaturization and unconventional materials, possibly exploiting biohybrid solutions (11). Soft-bodied robots enable alternative forms of interaction and physical self-organization that can be exploited in an innovative way. For instance, swarms of soft-bodied, biodegradable, millimeter-scale robots could be deployed in agricultural fields, coordinating to attack pests or harmful soil nematodes without damaging the crops, or in the sea to harvest microplastics by self-assembling into macroscopic structures to be later collected by larger water-cleaning robots. The last-and more challenging - frontier would be medical applications, requiring extreme miniaturization and biocompatibility. Considering the current advancements $(5,6)$, nanoscale robot swarms could be ready for testing by the mid-21st century. The successful deployment of robot swarms in these and other applications could pave the way for selforganized, bioinspired swarm robotics to become a mainstay of engineering in the second half of the century, possibly establishing itself as the standard way of designing complex robotic systems.

\section{REFERENCES}

1. C. R. Kube, H. Zhang, Collective robotics: From social insects to robots. Adapt. Behav. 2, 189-218 (1993).

2. O. H. Holland, C. Melhuish, Stigmergy, self-organization, and sorting in collective robotics. Artif. Life 5, 173-202 (1999).

3. S. Nouyan, R. Groß, M. Bonani, F. Mondada, M. Dorigo, Teamwork in self-organized robot colonies. IEEE Trans. Evol. Comput. 13, 695-711 (2009).

4. M. Dorigo, D. Floreano, L. M. Gambardella, F. Mondada S. Nolfi, T. Baaboura, M. Birattari, M. Bonani, M. Brambilla, A. Brutschy, D. Burnier, A. Campo, A. L. Christensen, A. Decugnière, G. Di Caro, F. Ducatelle, E. Ferrante, A. Förster, J. Guzzi, V. Longchamp, S. Magnenat, J. Martinez Gonzales, N. Mathews, M. M. de Oca, R. O'Grady, C. Pinciroli, G. Pini, P. Rétornaz, J. Roberts, V. Sperati, T. Stirling, A. Stranieri, T. Stützle, V. Trianni, E. Tuci, A. E. Turgut, F. Vaussard, Swarmanoid: A novel concept for the study of heterogeneous robotic swarms. IEEE Robot. Autom. Mag. 20, 60-71 (2013).

5. S. Jeon, S. Kim, S. Ha, S. Lee, E. Kim, S. Y. Kim, S. H. Park, J. H. Jeon, S. W. Kim, C. Moon, B. J. Nelson, J.-Y. Kim, S.-W. Yu, H. Choi, Magnetically actuated microrobots as a platform for stem cell transplantation. Sci. Robot. 4, eaav4317 (2019).

6. H. Xie, M. Sun, X. Fan, Z. Lin, W. Chen, L. Wang, L. Dong, Q. He, Reconfigurable magnetic microrobot swarm: Multimode transformation, locomotion, and manipulation. Sci. Robot. 4, eaav8006 (2019).

7. M. Rubenstein, A. Cornejo, R. Nagpal, Programmable self-assembly in a thousand-robot swarm. Science $\mathbf{3 4 5}$ 795-799 (2014)

8. I. Slavkov, D. Carrillo-Zapata, N. Carranza, X. Diego, F. Jansson, J. Kaandorp, S. Hauert, J. Sharpe, Morphogenesis in robot swarms. Sci. Robot. 3, eaau9178 (2018).

9. M. Birattari, A. Ligot, K. Hasselmann, Disentangling automatic and semi-automatic approaches to the optimization-based design of control software for robot swarms. Nat. Mach. Intell. 2, 494-499 (2020).

10. N. Mathews, A. L. Christensen, R. O'Grady, F. Mondada, $M$. Dorigo, Mergeable nervous systems for robots. Nat. Commun. 8, 439 (2017).

11. S. Kriegman, D. Blackiston, M. Levin, J. Bongard, A scalable pipeline for designing reconfigurable organisms. Proc. Natl. Acad. Sci. U.S.A. 117, 1853-1859 (2020).

10.1126/scirobotics.abe4385

Citation: M. Dorigo, G. Theraulaz, V. Trianni, Reflections on the future of swarm robotics. Sci. Robot. 5, eabe4385 (2020). 


\section{ScienceRobotics}

\section{Reflections on the future of swarm robotics}

Marco Dorigo, Guy Theraulaz and Vito Trianni

Sci. Robotics 5, eabe4385.

DOI: $10.1126 /$ scirobotics.abe4385

ARTICLE TOOLS

http://robotics.sciencemag.org/content/5/49/eabe4385

RELATED

http://robotics.sciencemag.org/content/robotics/4/30/eaav4317.full http://robotics.sciencemag.org/content/robotics/4/28/eaav8006.full http://robotics.sciencemag.org/content/robotics/3/25/eaau9178.full

REFERENCES

This article cites 11 articles, 2 of which you can access for free http://robotics.sciencemag.org/content/5/49/eabe4385\#BIBL

PERMISSIONS

http://www.sciencemag.org/help/reprints-and-permissions

Use of this article is subject to the Terms of Service

Science Robotics (ISSN 2470-9476) is published by the American Association for the Advancement of Science, 1200 New York Avenue NW, Washington, DC 20005. The title Science Robotics is a registered trademark of AAAS.

Copyright @ 2020 The Authors, some rights reserved; exclusive licensee American Association for the Advancement of Science. No claim to original U.S. Government Works 